\title{
Stability Comparison of IPMSM Sensorless Vector Control Systems Using Extended EMF
}

\author{
Mineo Tsuji, Hiroshi Mizusaki, Sin-ichi Hamasaki \\ Nagasaki University, Graduate School of Engineering \\ 1-14 Bunkyo-machi, Nagasaki, 852-8521, Japan
}

\begin{abstract}
Sensorless vector control of an interior permanent magnet synchronous motor (IPMSM) has been studied in many papers. The extended electromotive force (EMF) based method is one of the representative systems. We proposed a simplified method which estimates the rotor position using the controlled voltage without using observer. In this paper we have compared these methods through trajectories of poles and experimental transient responses for stability study.
\end{abstract}

Keywords- interior permanent magnet synchronous motor, sensorless vector control, extended EMF, stability analysis

\section{INTRODUCTION}

Because of high efficiency and small size, PMSM is used in many application. Especially, IPMSM is possible to produce high torque by using reluctance torque.

To realize a vector control of IPMSM, an encoder for detecting rotor position is used because it is necessary to control currents in accordance with the position of the rotor. However, there are problems; the cost of the position sensor, size of the apparatus and wiring of the signal line. Therefore, many sensorless control methods without using the position sensor are proposed ${ }^{[1]-[6]}$. The method using the extended EMF is one of the representative sensorless control methods. The extended EMF is estimated by a disturbance observer, and the positional information is obtained by using it ${ }^{[1][2]}$. On the other hand, we have proposed a simple sensorless method based on the extended $\mathrm{EMF}^{[7]}$. The rotor speed is estimated by the output voltage of the $d$-axis PI current controller with the non-interference control without using the disturbance observer. Although similar simplification is proposed in earlier papers ${ }^{[5][6]}$ for a non-salient pole PMSM, our proposed method can be applied to IPMSM by using the extended EMF.

In this paper, we discuss the stability by comparing the results of the proposed method with those of the conventional extended EMF sensorless one. The root loci of linear models, transient responses obtained by nonlinear models and experiments are demonstrated.

\section{SENSORLESS SYSTEMS}

\section{A. Extended EMF Model of IPMSM}

Voltage equation of IPMSM on $d-q$ rotating reference frame synchronized with the magnetic pole position is expressed as follows ${ }^{[1]}$ :

$$
\left[\begin{array}{c}
v_{d} \\
v_{q}
\end{array}\right]=\left[\begin{array}{cc}
R_{s}+p L_{d} & -\omega_{r} L_{q} \\
\omega_{r} L_{q} & R_{s}+p L_{d}
\end{array}\right]\left[\begin{array}{l}
i_{d} \\
i_{q}
\end{array}\right]+\left[\begin{array}{c}
0 \\
E_{e x}
\end{array}\right]
$$

where,

$$
E_{e x}=\omega_{r}\left\{\left(L_{d}-L_{q}\right) i_{d}+\psi\right\}-\left(L_{d}-L_{q}\right) p i_{q}
$$

By converting the reference frame which rotates at an angular speed $\hat{\omega}$ as shown in Fig.1, the following equation can be obtained from (1):

$$
\left[\begin{array}{c}
v_{\gamma} \\
v_{\delta}
\end{array}\right]=\left[\begin{array}{cc}
R_{s}+p L_{d} & -\omega_{r} L_{q} \\
\omega_{r} L_{q} & R_{s}+p L_{d}
\end{array}\right]\left[\begin{array}{l}
i_{\gamma} \\
i_{\delta}
\end{array}\right]+\left[\begin{array}{l}
e_{\gamma} \\
e_{\delta}
\end{array}\right]
$$

The second term of the right side is called the extended EMF and expressed as

$$
\left[\begin{array}{c}
e_{\gamma} \\
e_{\delta}
\end{array}\right]=E_{e x}\left[\begin{array}{c}
\sin \theta_{e} \\
\cos \theta_{e}
\end{array}\right]+\left(\hat{\omega}-\omega_{r}\right) L_{d}\left[\begin{array}{c}
-i_{\delta} \\
i_{\gamma}
\end{array}\right]
$$

\section{B. Conventional Method}

Conventional sensorless vector control system is shown in Fig. $2^{[1][2]}$. The extended EMF is estimated by using disturbance observer as follows;

$$
\begin{aligned}
& \hat{e}_{\gamma}=\frac{g}{s+g}\left\{v_{\gamma}^{*}+\hat{\omega} L_{q} i_{\delta}-\left(R_{s}+s L_{d}\right) i_{\gamma}\right\} \\
& \hat{e}_{\delta}=\frac{g}{s+g}\left\{v_{\delta}^{*}-\hat{\omega} L_{q} i_{\gamma}-\left(R_{s}+s L_{d}\right) i_{\delta}\right\}
\end{aligned}
$$

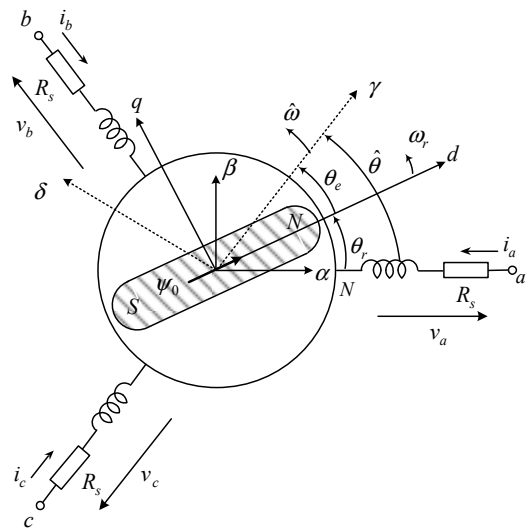

Fig. 1. Model of IPMSM. 
From (4), the magnetic pole position error $\hat{\theta}_{e}$ is computed by the following equation:

$$
\hat{\theta}_{e}=\tan ^{-1}\left(\frac{\hat{e}_{\gamma}}{\hat{e}_{\delta}}\right)
$$

Using (7), the rotor angular speed is estimated by

$$
\hat{\omega}=-\left(K_{e p}+\frac{K_{e i}}{s}\right) \hat{\theta}_{e}
$$

By using damping coefficient $\zeta$ and natural angular frequency $\omega_{n}$, the PI speed estimation gains of Fig.3 are designed as follows ${ }^{[2]}$ :

$$
K_{e p}=2 \zeta \omega_{n}, K_{e i}=\omega_{n}^{2}
$$

To reduce the influence of noise, the rotor angular speed is estimated using a low-pass filter as follows:

$$
\hat{\omega}_{r}=\frac{\omega_{c}}{s+\omega_{c}} \hat{\omega}
$$

The pole position is computed by the following equation.

$$
\hat{\theta}=\frac{1}{s} \hat{\omega}
$$

By considering the difference between the $\gamma-\delta$ axis and $d-q$ axis, the actual position estimation error $\theta_{e}$ can be expressed as follows from Fig.1:

$$
\theta_{e}=\hat{\theta}-\theta_{r}
$$

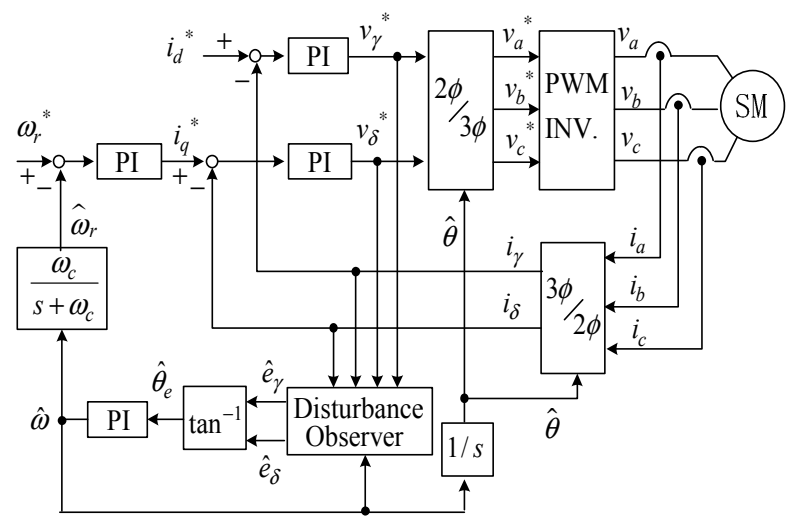

Fig.2. Conventional Sensorless Vector Control System.

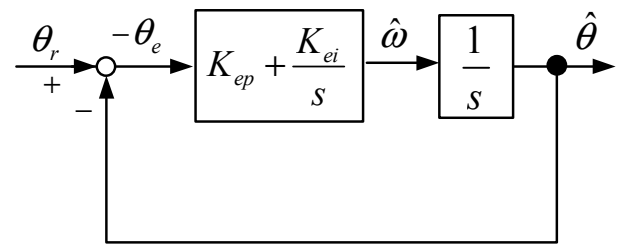

Fig.3. Approximated block diagram for speed estimation.

\section{Proposed Method}

Proposed sensorless vector control system is shown in Fig. $4^{[7]}$

From (4), when the error of the magnetic pole position is small, the following equation is obtained by approximating $\sin \theta_{e} \simeq \theta_{e}$ and $\cos \theta_{e} \simeq 1$.

$$
\left[\begin{array}{l}
e_{\gamma} \\
e_{\delta}
\end{array}\right] \simeq E_{e x}\left[\begin{array}{c}
\sin \theta_{e} \\
\cos \theta_{e}
\end{array}\right] \simeq E_{e x}\left[\begin{array}{c}
\theta_{e} \\
1
\end{array}\right]
$$

From (13), the position error $\theta_{e}$ is approximated as

$$
\theta_{e} \simeq \frac{e_{\gamma}}{E_{e x}}
$$

Therefore, it is possible to estimate the position error by using $e_{\gamma}$.

In Fig. 4 , the $d$-axis PI current control is given by the following equation.

$$
e_{\gamma}^{*}=\left(K_{p d}+\frac{K_{i d}}{s}\right)\left(i_{d}^{*}-i_{\gamma}\right)
$$

The $\gamma$ - axis voltage reference $v_{\gamma}^{*}$ is calculated by the following equation ignoring the differential term of (3).

$$
v_{\gamma}^{*}=R_{s}^{*} i_{d}^{*}-L_{q}^{*} \hat{\omega}_{r} i_{\delta}+e_{\gamma}^{*}
$$

The rotor angular speed $\hat{\omega}$ is estimated by the following equation using the output voltage $e_{\gamma}^{*}$ from (14).

$$
\hat{\omega}=-\left(K_{e p}+\frac{K_{e i}}{s}\right) \frac{e_{\gamma}^{*}}{E_{e x}^{*}}
$$

$E_{e x}^{*}$ is changed as a function of speed by neglecting differential term of (2).

$$
E_{e x}^{*}=\hat{\omega}_{r}\left\{\left(L_{d}-L_{q}\right) i_{d}^{*}+\psi\right\}
$$

By using damping coefficient $\zeta$ and natural angular frequency $\omega_{n}$, the PI control gains of (17) are designed as same as (9). Figure 3 shows also an equivalent block diagram for proposed speed estimation shown in Fig.4.

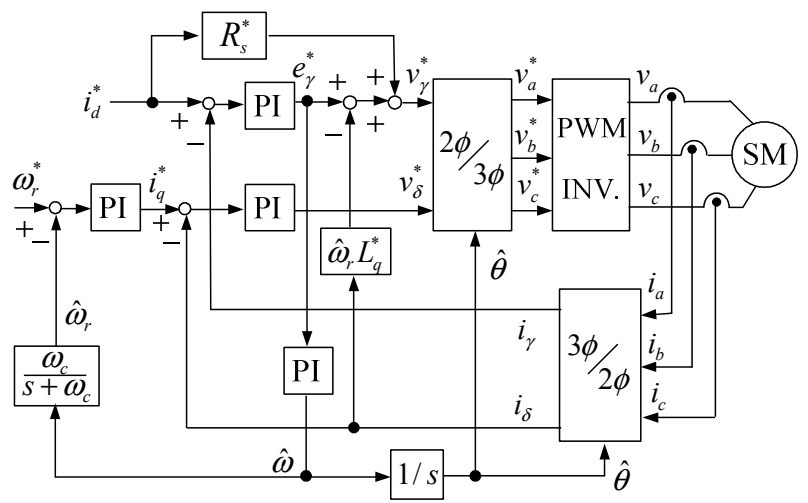

Fig.4. Proposed sensorless vector control system.

\section{STABILITY ANALYSIS}

\section{A. Nonlinear Model}

In order to analyze the system shown in Fig.2, we choose the $\gamma-\delta$ axis of Fig.1. By assuming ideal voltage control of PWM inverter, we have

$$
v_{a}=v_{a}^{*}, v_{b}=v_{b}^{*}, v_{c}=v_{c}^{*}
$$

Since $\gamma \delta$ transformation of controller is the same as the analysis, the following relation is obtained from (19).

$$
v_{\gamma}=v_{\gamma}^{*}, v_{\delta}=v_{\delta}^{*}
$$

By using the actual error angle $\theta_{e}$, the $d-q$ variables are expressed by the following co-ordinate transformation. 


$$
\begin{aligned}
& {\left[\begin{array}{l}
v_{d} \\
v_{q}
\end{array}\right]=\left[\begin{array}{cc}
\cos \theta_{e} & -\sin \theta_{e} \\
\sin \theta_{e} & \cos \theta_{e}
\end{array}\right]\left[\begin{array}{c}
v_{\gamma}^{*} \\
v_{\delta}^{*}
\end{array}\right]} \\
& {\left[\begin{array}{l}
i_{d} \\
i_{q}
\end{array}\right]=\left[\begin{array}{cc}
\cos \theta_{e} & -\sin \theta_{e} \\
\sin \theta_{e} & \cos \theta_{e}
\end{array}\right]\left[\begin{array}{l}
i_{\gamma} \\
i_{\delta}
\end{array}\right]}
\end{aligned}
$$

The $d-q$ state equations of IPMSM are obtained by Park's equation as follows:

$$
\begin{aligned}
& p i_{d}=\frac{1}{L_{d}}\left(v_{d}-R_{s} i_{d}+\omega_{r} L_{q} i_{q}\right) \\
& p i_{q}=\frac{1}{L_{q}}\left(v_{q}-R_{s} i_{q}-\omega_{r} L_{d} i_{d}-\omega_{r} \psi\right) \\
& p \omega_{r}=\frac{P^{2}}{4 J}\left\{\psi i_{q}+\left(L_{d}-L_{q}\right) i_{d} i_{q}\right\}-\frac{P}{2 J} T_{L}
\end{aligned}
$$

where, $P$ is number of poles, $J$ is moment of inertia, and $T_{L}$ is load torque.

The disturbance observer is described as follows:

$$
\begin{aligned}
& \hat{e}_{\gamma}=g z_{1}-g L_{d} i_{\gamma} \\
& \text { where, } z_{1}=\frac{1}{s+g}\left(v_{\gamma}^{*}+\hat{\omega} L_{q} i_{\delta}-R_{s} i_{\gamma}+g L_{d} i_{\gamma}\right) \\
& \hat{e}_{\delta}=g z_{2}-g L_{d} i_{\delta} \\
& \text { where, } z_{2}=\frac{1}{s+g}\left(v_{\delta}^{*}-\hat{\omega} L_{q} i_{\gamma}-R_{s} i_{\delta}+g L_{d} i_{\delta}\right)
\end{aligned}
$$

The PI controllers and low pass filter are expressed by the following equations.

PI speed controller:

$$
\begin{aligned}
& p w_{1}=\omega_{r}{ }^{*}-\hat{\omega}_{r} \\
& i_{q}{ }^{*}=K_{p s}\left(\omega_{r}{ }^{*}-\hat{\omega}_{r}\right)+K_{i s} w_{1}
\end{aligned}
$$

$D$ axis PI controller:

$$
\begin{aligned}
& p w_{2}=i_{d}{ }^{*}-i_{\gamma} \\
& v_{\gamma}{ }^{*}=K_{p d}\left(i_{d}{ }^{*}-i_{\gamma}\right)+K_{i d} w_{2}
\end{aligned}
$$

$Q$ axis PI controller:

$$
\begin{aligned}
& p w_{3}=i_{q}^{*}-i_{\delta} \\
& v_{\delta}^{*}=K_{p q}\left(i_{q}^{*}-i_{\delta}\right)+K_{i q} w_{3}
\end{aligned}
$$

PI speed estimator:

$$
\begin{aligned}
& p z_{3}=-\hat{\theta}_{e} \\
& \hat{\omega}=-K_{e p} \hat{\theta}_{e}+K_{e i} z_{3}
\end{aligned}
$$

Low pass filter of speed estimation:

$$
p \hat{\omega}_{r}=-\omega_{c} \hat{\omega}_{r}+\omega_{c} \hat{\omega}
$$

By taking the derivative of (12), we have

$$
p \theta_{e}=\hat{\omega}-\omega_{r}
$$

A nonlinear model of the sensorless system shown in Fig. 2 has been obtained. By using this model, we can compute transient responses.

The steady-state values are obtained by setting $p=0$ or $s=0$ in the nonlinear model except for (11). From (36), we have

$$
\hat{\theta}_{e 0}=0
$$

Then, $\hat{e}_{r}$ is obtained from (7) as

$$
\hat{e}_{\gamma 0}=0
$$

When the motor resistance and inductance are equal to those actual values, the following equation is obtained.

$$
\theta_{e 0}=0
$$

In this case, the $\gamma-\delta$ axis coincides with the $d-q$ axis.

B. Linear Model

By considering a small variation in the vicinity of the equilibrium point of nonlinear differential equations, a linear model is obtained.

A linear model of the IPMSM on $d-q$ rotating reference frame can be expressed as follows ${ }^{[7]}$ :

$p \Delta \boldsymbol{x}_{s}=\boldsymbol{A}_{s} \Delta \boldsymbol{x}_{s}+\boldsymbol{B} \Delta \boldsymbol{u}_{s}+\boldsymbol{B}_{T} \Delta T_{L}$

where, $\Delta \boldsymbol{x}_{s}=\left[\begin{array}{lll}\Delta i_{d} & \Delta i_{q} & \Delta \omega_{r}\end{array}\right]^{T}, \Delta \boldsymbol{u}_{s}=\left[\begin{array}{ll}\Delta v_{d} & \Delta v_{q}\end{array}\right]^{T}$

By taking small perturbation of (21) and (22), we have

$$
\begin{aligned}
& \Delta v_{d}=\Delta v_{\gamma}^{*}-v_{q 0} \Delta \theta_{e} \\
& \Delta v_{q}=\Delta v_{\delta}^{*}+v_{d 0} \Delta \theta_{e} \\
& \Delta i_{\gamma}=\Delta i_{d}+i_{q 0} \Delta \theta_{e} \\
& \Delta i_{\delta}=\Delta i_{q}-i_{d 0} \Delta \theta_{e}
\end{aligned}
$$

From (7), the following equation is obtained.

$$
\Delta \hat{\theta}_{e}=\frac{\hat{e}_{\delta 0}}{\hat{e}_{\delta 0}^{2}+\hat{e}_{\gamma 0}^{2}} \Delta \hat{e}_{\gamma}-\frac{\hat{e}_{\gamma 0}}{\hat{e}_{\delta 0}^{2}+\hat{e}_{\gamma 0}^{2}} \Delta \hat{e}_{\delta}
$$

By using (41), we have

$$
\Delta \hat{\theta}_{e}=\frac{1}{\hat{e}_{\delta 0}} \Delta \hat{e}_{\gamma}
$$

A linear model of the controller of Fig. 2 is expressed as follows(refer to Appendix):

$$
p \Delta \boldsymbol{w}=\boldsymbol{A}_{w} \Delta \boldsymbol{w}+\boldsymbol{A}_{x} \Delta \boldsymbol{x}_{s}+\boldsymbol{B}_{r} \Delta \boldsymbol{r}
$$

where ,

$$
\begin{aligned}
& \Delta \boldsymbol{w}=\left[\begin{array}{llllllll}
\Delta z_{1} & \Delta z_{2} & \Delta z_{3} & \Delta \hat{\omega}_{r} & \Delta w_{1} & \Delta w_{2} & \Delta w_{3} & \Delta \theta_{e}
\end{array}\right]^{T}, \\
& \Delta \boldsymbol{r}=\left[\begin{array}{lll}
\Delta i_{d}{ }^{*} & \Delta \omega_{r}{ }^{*}
\end{array}\right]^{T}
\end{aligned}
$$

The $\Delta \boldsymbol{w}$ is state vector. $\Delta \mathrm{z}_{1}$ and $\Delta z_{2}$ for disturbance observer, $\Delta z_{3}$ for speed estimation, $\Delta w_{1}$ for speed PI control, $\Delta w_{2}$ and $\Delta w_{3}$ for current PI control are necessary.

The relationship between the controller and the motor input is expressed as follows (see Appendix):

$$
\Delta \boldsymbol{u}_{s}=\boldsymbol{F}_{w} \Delta \boldsymbol{w}+\boldsymbol{F}_{\boldsymbol{x}} \Delta \boldsymbol{x}_{s}+\boldsymbol{F}_{\boldsymbol{r}} \Delta \boldsymbol{r}
$$

From (43), (50) and (51), the linear model of overall system is derived as follows:

$$
\begin{aligned}
p\left[\begin{array}{c}
\Delta \boldsymbol{x}_{s} \\
\Delta \boldsymbol{w}
\end{array}\right]= & {\left[\begin{array}{cc}
\boldsymbol{A}_{s}+\boldsymbol{B}_{s} \boldsymbol{F}_{x} & \boldsymbol{B}_{s} \boldsymbol{F}_{w} \\
\boldsymbol{A}_{\boldsymbol{x}} & \boldsymbol{A}_{w}
\end{array}\right]\left[\begin{array}{l}
\Delta \boldsymbol{x}_{s} \\
\Delta \boldsymbol{w}
\end{array}\right] } \\
& +\left[\begin{array}{c}
\boldsymbol{B}_{s} \boldsymbol{F}_{r} \\
\boldsymbol{B}_{r}
\end{array}\right] \Delta \boldsymbol{r}+\left[\begin{array}{c}
\boldsymbol{B}_{T} \\
\mathbf{0}
\end{array}\right] \Delta T_{L}
\end{aligned}
$$

As for the proposed method shown in Fig.4, a linear model is derived in the same manner ${ }^{[7]}$.

Using the linear models described above, the stability analysis is performed by computing the eigenvalues of system matrix. The tested IPMSM has the following rated and nominal values: rated output $800 \mathrm{~W}$, rated speed $3000 \mathrm{rpm}, P=8, R_{s}=0.4 \Omega, L_{d}=3.42 \mathrm{mH}, L_{q}=$ $3.82 \mathrm{mH}, \psi=0.0845 \mathrm{~Wb}$ and $J=0.0048 \mathrm{kgm}^{2}$. We discuss the effect of the variable parameters which are the 
damping coefficient $\zeta$, the natural angular frequency $\omega_{n}$ of the PI speed estimator and the cross angular frequency $\omega_{s c}$ of the PI speed controller. In this case, the speed reference $N_{r}^{*}=500 \mathrm{rpm}$, the cut off frequency of LPF $\omega_{c}=300 \mathrm{rad} / \mathrm{s}$, the load torque $T_{L}=0.6 \mathrm{Nm}$. The observer gain of the conventional system is set as $g=600 \mathrm{rad} / \mathrm{s}$. The cut-off frequencies of all PI current controllers are designed 1000 rad/s.

Fig.5 shows the trajectories of poles for changes of $\omega_{s c}$ and $\omega_{n}$. The root loci of the conventional method and the proposed method are shown in (a) and (b) respectively. From Fig.5, it is observed that the system becomes oscillating when $\omega_{n}$ is small and $\omega_{s c}$ is large. By comparing the root loci of the proposed method and those of the conventional method, it is found that the dominant root loci are very close. Fig.6 shows the trajectories of poles for changes of $\omega_{s c}$ and $\zeta$. From Fig.6, the system becomes unstable when $\zeta$ is small and $\omega_{s c}$ is large. The dominant root loci are also very close for the conventional method and the proposed one.

Fig.7 shows the root loci when the pole position is estimated by the output of the LPF without using (11) in the conventional method as

$$
\hat{\theta}=\hat{\omega}_{r} / s
$$

By comparing the root loci of Fig.5(a) and those of Fig.7, the difference occurs when $\omega_{n}$ is large. In Fig.7, the system becomes unstable when $\omega_{n}$ is large because of LPF. In a real system, a delay occurs in $\hat{\theta}$ for per- forming PWM control by a DSP. Consequently, it is considered

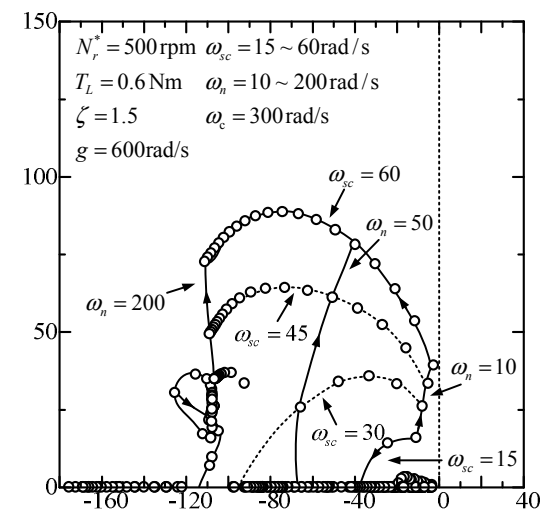

(a) Conventional method

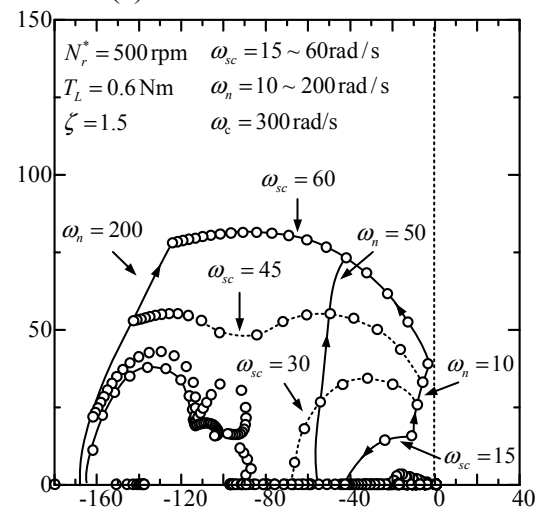

(b) Proposed method

Fig.5. Trajectories of poles for changes of $\omega_{s c}$ and $\omega_{n}$.

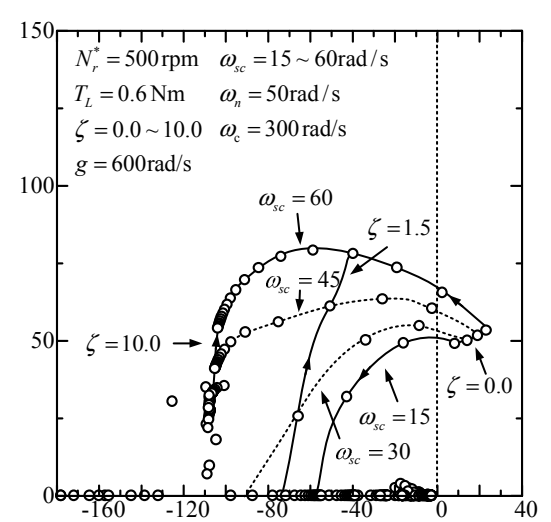

(a) Conventional method

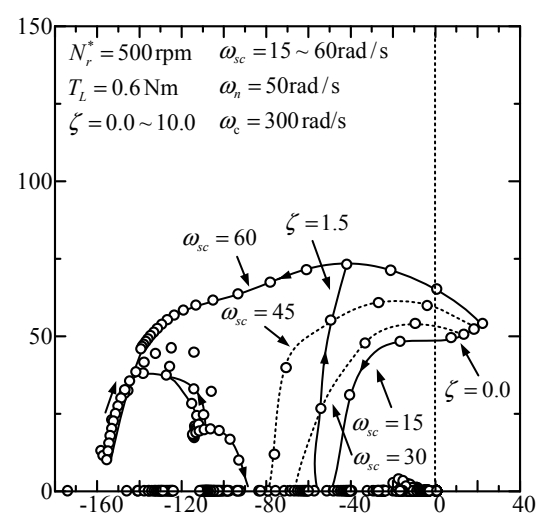

(b) Proposed method

Fig.6. Trajectories of poles for changes of $\omega_{s c}$ and $\zeta$.

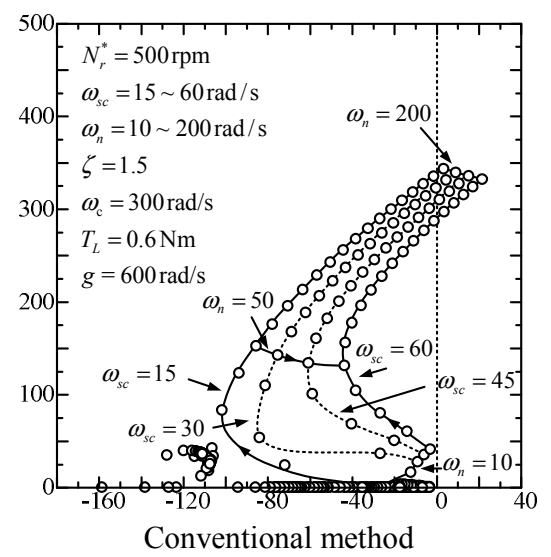

Fig.7. Trajectories of poles for changes of $\omega_{s c}$ and $\omega_{n}$ when $\hat{\theta}$ is computed by $\hat{\omega}_{r}$.

that there is a limit of $\omega_{n}$ as shown in Fig.7 in practical system even if we use (11).

\section{SimUlation AND EXPERIMENTAL RESULTS}

With the control parameters that we used in the stability analysis, we simulated the transient responses for the step change of speed reference from 500rpm to $550 \mathrm{rpm}$ by the non-linear models. In addition, we performed the experiment at the same conditions.

Simulation results are shown in Figs.8, 10, 12, 14 and 16. Corresponding experimental results are shown in Figs.9, 11, 13, 15 and 17. The parameters $\omega_{s c}$ is fixed 
and $\omega_{n}$ and $\zeta$ are changed. $N_{r}$ is the actual speed, $\hat{N}_{r}$ is the estimated speed, $\theta_{e}$ is the magnetic pole position error.

In Fig.8, the oscillation of low frequency is observed in both methods and the position error $\theta_{e}$ is large. In Fig.9, the experimental results are similar to the simulation results. When $\omega_{n}=12$, the dominant pole is close to the imaginary axis from Fig.5. Therefore, it is considered that the oscillation is observed. If $\omega_{n}$ in less than 12 , the system becomes unstable experimentally. When $\omega_{n}=50$, the root is stabilized in Fig.5, the oscillation such as Fig.6 disappears in Fig.10. The experimental results of Fig.11 are in good agreement with the simulation results except for the high frequency ripples. Fig.12 is the case where $\omega_{n}$ is further increased. Speed estimation gain is increased, the position error becomes considerably small. In the experimental results of Fig.13, the ripples of high frequencies are observed in $\hat{N}_{r}$ and $\theta_{e}$. However, these values are processed only in the DSP and high frequency ripple of the actual speed $N_{r}$ which is important for the application is small. From Figs. (14) - (17) show the results for the change of $\zeta$. It is confirmed that the system becomes oscillating or unstable when $\zeta$ is small.

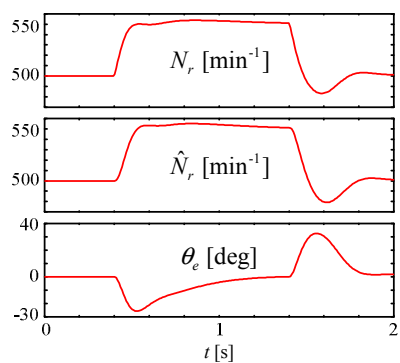

(a)Conventional method

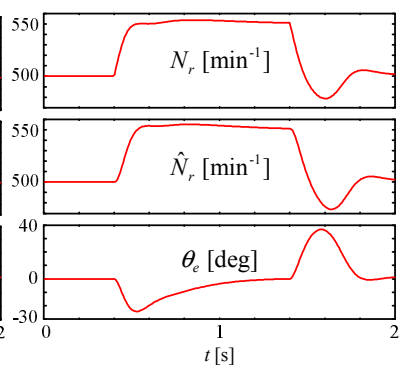

(b)Proposed method
Fig.8. Simulation Results $\left(\omega_{n}=12, \zeta=1.5, \omega_{s c}=15\right)$.

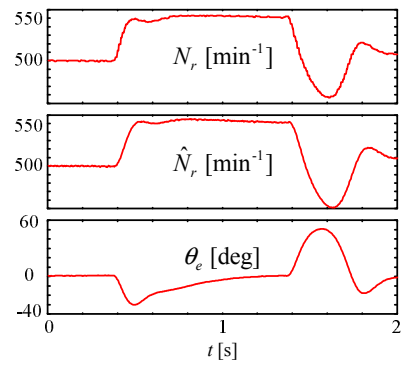

(a)Conventional method

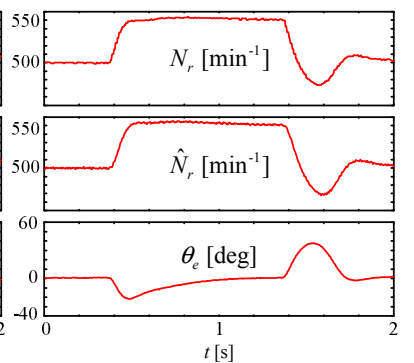

(b)Proposed method
Fig.9. Experimental results $\left(\omega_{n}=12, \zeta=1.5, \omega_{s c}=15\right)$.

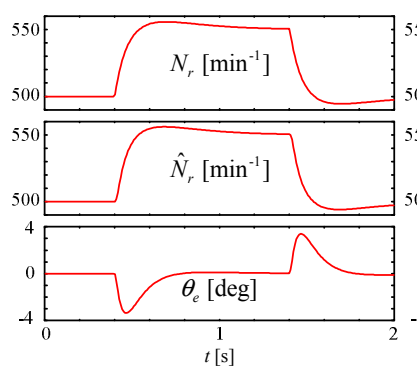

(a)Conventional method

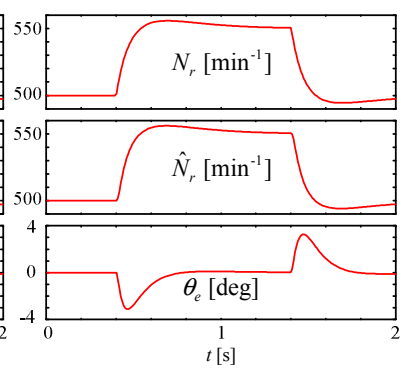

(b)Proposed method
Fig.10. Simulation results $\left(\omega_{n}=50, \zeta=1.5, \omega_{s c}=15\right)$.

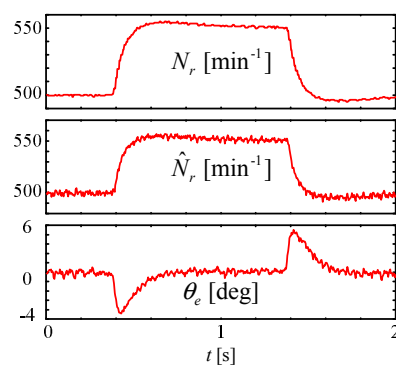

(a)Conventional method
(b)Proposed method

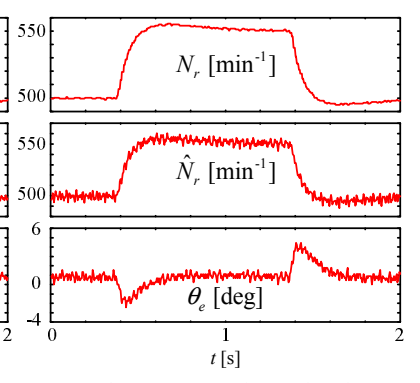

Fig.11. Experimental reults $\left(\omega_{n}=50, \zeta=1.5, \omega_{s c}=15\right)$.

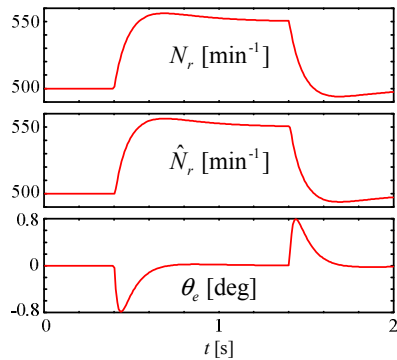

(a)Conventional method

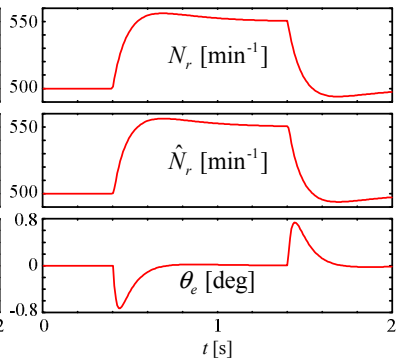

(b)Proposed method
Fig.12. Simulation results $\left(\omega_{n}=120, \zeta=1.5, \omega_{s c}=15\right)$.

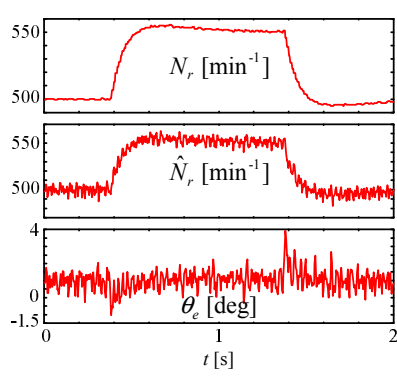

(a)Conventional method

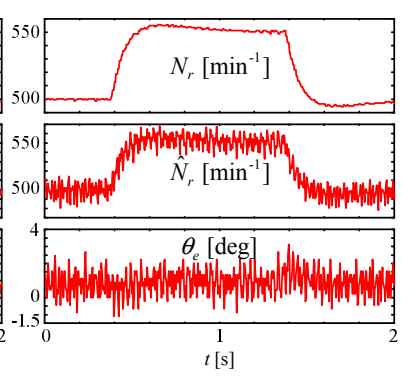

(b)Proposed method
Fig.13. Experimental results $\left(\omega_{n}=120, \zeta=1.5, \omega_{s c}=15\right)$.

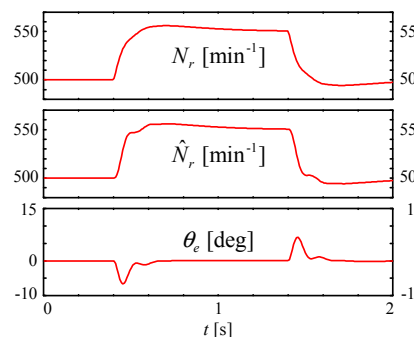

(a)Conventional method

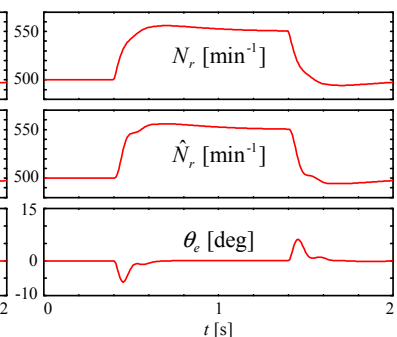

(b)Proposed method
Fig.14. Simulation results $\left(\omega_{n}=50, \zeta=0.5, \omega_{s c}=15\right)$.

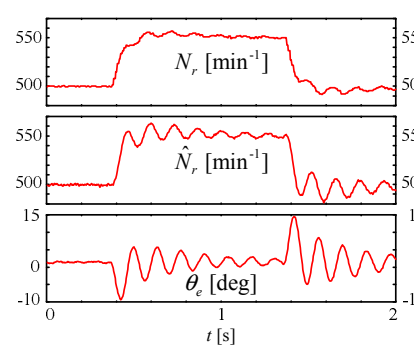

(a)Conventional method

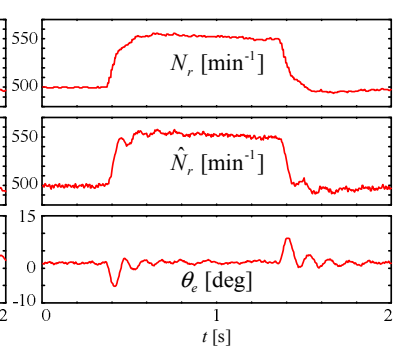

(b)Proposed method
Fig.15. Experimental results $\left(\omega_{n}=50, \zeta=0.5, \omega_{s c}=15\right)$. 


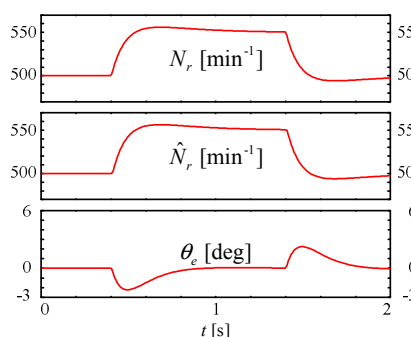

(a)Conventional method

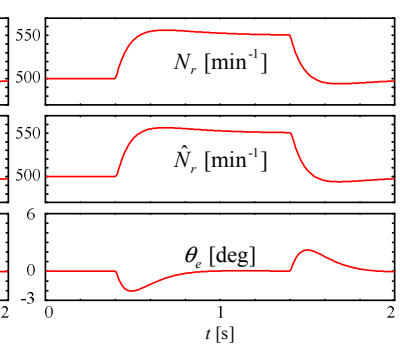

(b)Proposed method

Fig.16. Simulation results $\left(\omega_{n}=50, \zeta=3.0, \omega_{s c}=15\right)$.

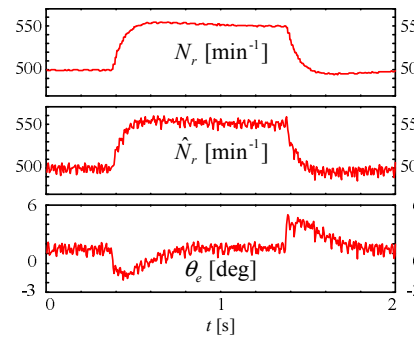

(a)Conventional method

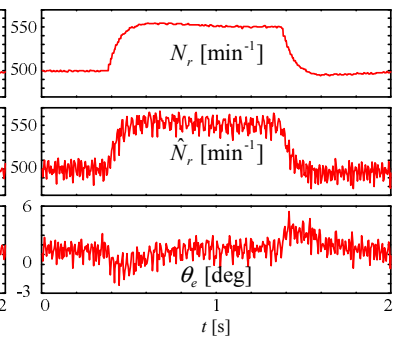

(b)Proposed method
Fig.17. Experimental results $\left(\omega_{n}=50, \zeta=3.0, \omega_{s c}=15\right)$.

Conventional method improves high frequency ripples of estimated speed by the help of disturbance observer. However, the ripples of actual speed which is important for the applications are almost same. The cut-off frequencies of all PI current controllers are designed $1000 \mathrm{rad} / \mathrm{s}$. The influence of this frequency for proposed method is not sensitive.

\section{CONCLUSIONS}

For IPMSM sensorless vector control using the extended EMF, we have compared the proposed method with the conventional method by the root loci, non-linear simulation and experiment. Concerning these points, the results of the proposed method is almost the same as those of the conventional method. Therefore, it is concluded that the proposed method is useful because of its simpler structure.

\section{REFERENCES}

[1] Z. Chen, M. Tomita, S. Doki, and S. Okuma, "An Extended Electromotive Force Model for Sensorless Control of Interior Permanent-Magnet Synchronous Motors," IEEE Trans. Ind. Electron, vol.50, no.2, pp.288-295, 2003.

[2] S. Morimoto, K. Kawamoto, M. Sanada, and Y. Takeda, "Sensorless Control Strategy for Salient-Pole PMSM Based on Extended EMF in Rotating Reference Frame," IEEE Trans. Ind. Applicat, vol.38, no4, pp.1054-1061, 2002.

[3] K. Sakamoto, Y. Iwaji, T. Endo, T. Taniguchi, T. Niki, M. Kawamata, and A. Kawamura, "Position Sensorless Vector Control of Permanent Magnet Synchronous Motors for Electrical Household Appliances," Proc. of PCC-Nagoya 2007, LS4-3-3, pp.1119-1125, 2007.

[4] K. Yamanaka, T. Ohnishi, and M. Hojo, "A Novel Position Sensorless Vector Control of Permanent-Magnet Synchronous Motors," Proc. of PCC-Nagoya 2007, DS8-3-1, pp.290-295, 2007.

[5] B. H. Bae, S. K. Sul, J. H. Kwon and J.S. Byeon, "Implementation of Sensorless Vector Control for Super-High-Speed PMSM of Turbo-Compressor," IEEE Trans. Ind. Appl., vol.39, no3, pp.811$818,2003$.
[6] J. K. Seok, J. K. Lee, and D.C. Lee, "Sensorless Speed Control of Nonsalient Permanent-Magnet Synchronous Motor Using RotorPosition-Ttracking PI Controller," IEEE Trans. Ind. Electron, vol.53, no2, pp.399-405, 2006.

[7] M. Tuji, K. Kojima, G. Mangindaan, D. Akafuji, S. Hamasaki, "Stability Study of a Permanent Magnet Synchronous Motor Sensorless Vector Control System Based on Extended EMF Model," IEEJ Journal of Industry Applications, vol.1 no.3 pp.148-154, 2012 .

\section{APPENDIX}

$$
\begin{aligned}
& \boldsymbol{A}_{w}=\left[\begin{array}{ccccc}
-g-k_{1} i_{\delta 0} & 0 & L_{q} i_{\delta 0} K_{e i} & 0 & 0 \\
k_{1} i_{\gamma 0} & -g & -L_{q} i_{\gamma 0} K_{e i} & -K_{p q} K_{p s} & K_{p q} K_{i s} \\
-\frac{g}{\hat{e}_{\delta 0}} & 0 & 0 & 0 & 0 \\
-\frac{g \omega_{c} K_{e p}}{\hat{e}_{\delta 0}} & 0 & \omega_{c} K_{e i} & -\omega_{c} & 0 \\
0 & 0 & 0 & -1 & 0 \\
0 & 0 & 0 & 0 & 0 \\
0 & 0 & 0 & -K_{p s} & K_{i s} \\
-\frac{K_{e p} g}{\hat{e}_{\delta 0}} & 0 & K_{e i} & 0 & 0
\end{array}\right. \\
& K_{i d} \quad 0 \quad k_{3} i_{q 0} i_{\delta 0}-\hat{\omega}_{0} L_{q} i_{d 0}+k_{4} i_{q 0} \\
& 0 \quad K_{i q} \quad-k_{3} i_{q 0} i_{\gamma 0}-\hat{\omega}_{0} L_{q} i_{q 0}-k_{5} i_{d 0} \\
& 0 \quad 0 \quad \frac{g L_{d} i_{q 0}}{\hat{e}_{\delta 0}} \\
& \text { * } 0 \quad 0 \quad k_{2} \omega_{c} i_{q 0} \\
& \begin{array}{lll}
0 & 0 & 0
\end{array} \\
& \begin{array}{lll}
0 & 0 & -i_{q 0}
\end{array} \\
& \begin{array}{lll}
0 & 0 & i_{d 0}
\end{array} \\
& \begin{array}{lll}
0 & 0 & k_{2} i_{q 0}
\end{array}
\end{aligned}
$$

where, $k_{1}=\frac{L_{q} K_{e p} g}{\hat{e}_{\delta 0}}, k_{2}=\frac{L_{d} K_{e p} g}{\hat{e}_{\delta 0}}, k_{3}=\frac{L_{d} L_{q} K_{e p} g}{\hat{e}_{\delta 0}}$

$$
k_{4}=g L_{d}-R_{s}-K_{p d}, k_{5}=g L_{d}-R_{s}-K_{p q}
$$$$
\boldsymbol{A}_{x}=\left[\begin{array}{ccc}
k_{3} i_{\delta 0}+k_{4} & \hat{\omega}_{0} L_{q} & 0 \\
-k_{3} i_{\gamma 0}-\hat{\omega}_{0} L_{q} & k_{5} & 0 \\
g L_{d} / \hat{e}_{\delta 0} & 0 & 0 \\
k_{2} \omega_{c} & 0 & 0 \\
0 & 0 & 0 \\
-1 & 0 & 0 \\
0 & -1 & 0 \\
k_{2} & 0 & -1
\end{array}\right], \boldsymbol{B}_{r}=\left[\begin{array}{cc}
K_{p d} & 0 \\
0 & K_{p q} K_{p s} \\
0 & 0 \\
0 & 0 \\
0 & 1 \\
1 & 0 \\
0 & K_{p s} \\
0 & 0
\end{array}\right]
$$

$$
\boldsymbol{F}_{w}=\left[\begin{array}{cllccccc}
0 & 0 & 0 & 0 & 0 & K_{i d} & 0 & -v_{q 0}-K_{p d} i_{q 0} \\
0 & 0 & 0-K_{p q} K_{p s} & K_{p q} K_{i s} & 0 & K_{i q} & v_{d 0}+K_{p q} i_{d o}
\end{array}\right]
$$$$
\boldsymbol{F}_{x}=\left[\begin{array}{ccc}
-K_{p d} & 0 & 0 \\
0 & -K_{p q} & 0
\end{array}\right]
$$$$
\boldsymbol{F}_{r}=\left[\begin{array}{cc}
K_{p d} & 0 \\
0 & K_{p q} K_{p s}
\end{array}\right]
$$ 